\title{
Status of Emergency Department Seventy-Two Hour Return Visits Among Homeless Patients
}

\author{
Heidi Knowles ${ }^{\mathrm{a}}$, Charles Huggins ${ }^{\mathrm{a}}$, Richard D. Robinson ${ }^{\mathrm{a}}$, Rosalia Mbugua ${ }^{\mathrm{a}}$, \\ Jessica Laureano-Phillips ${ }^{\mathrm{a}}$, Shrunjal M. Trivedib, Jessica Kirby ${ }^{\mathrm{a}}$, \\ Nestor R. Zenarosa ${ }^{\mathrm{a}}$, Hao Wang ${ }^{\mathrm{a}, \mathrm{c}}$
}

\begin{abstract}
Background: We aim to externally validate the status of emergency department (ED) appropriate utilization and 72-h ED returns among homeless patients.

Methods: This is a retrospective single-center observational study. Patients were divided into two groups (homeless versus non-homeless). Patients' general characteristics, clinical variables, ED appropriate utilization, and ED return disposition deviations were compared and analyzed separately.
\end{abstract}

Results: Study enrolled a total of 63,990 ED visits. Homeless patients comprised $9.3 \%(5,926)$ of visits. Higher ED 72-h returns occurred among homeless patients in comparison to the non-homeless patients $(17 \%$ versus $5 \%, \mathrm{P}<0.001)$. Rate of significant $\mathrm{ED}$ disposition deviations (e.g., admission, triage to operation room, or death) on return visits were lower in homeless patients when compared to non-homeless patient populations ( $15 \%$ versus $23 \%, \mathrm{P}<0.001)$.

Conclusions: Though ED return rate was higher among homeless patients, return visit case management seems appropriate, indicating that 72-h ED returns might not be an optimal healthcare quality measurement for homeless patients.

Keywords: Emergency department; Homeless; Appropriate utilization; ED return visit

\section{Introduction}

Patients discharged from the emergency department (ED)

Manuscript submitted January 9, 2019, accepted January 28, 2019

aDepartment of Emergency Medicine, Integrative Emergency Services, John Peter Smith Health Network, 1500 S. Main St., Fort Worth, TX 76104, USA ${ }^{b}$ Department of Family Medicine, Texas College of Osteopathic Medicine, University of North Texas Health Science Center, 3500 Camp Bowie Blvd, Fort Worth, TX 76107, USA

${ }^{\mathrm{c}}$ Corresponding Author: Hao Wang, John Peter Smith Health Network, 1500 S. Main St., Fort Worth, TX 76104, USA. Email: hwang01@jpshealth.org

doi: https://doi.org/10.14740/jocmr3747 and return within $72 \mathrm{~h}$ from the index ED visit are considered high-risk relative to increased morbidity and mortality $[1,2]$. Therefore, 72-h ED return visits (EDRVs) are considered an appropriate patient care quality measurement [3]. Studies in recent years show no significant differences in terms of hospital admissions, ED management, and medical errors among 72-h EDRV patients in comparison to others thus raising the question of whether EDRV is a meaningful patient care quality marker of ED operations [4-6]. Current studies further differentiate EDRV based on different patient populations (e.g., geriatrics) and disease patterns (e.g., patients with traumatic brain injuries, abdominal pain, etc.) and show diversity of risks predictive of such returns along with potential patient care outcome risks relative to certain patient populations [7-9]. This suggests EDRV might reflect patient care quality within subgroup populations.

Homeless patients are considered a special patient population in regard to high-risk for hospital admission and mortality when experiencing early EDRV $[10,11]$. Special attention to unique needs and interventions to mitigate negative effects of these are made available to this patient population as a means to decrease ED utilization and improve patient care quality [12, 13]. Despite various interventions (e.g., arranging primary care physician follow-up, providing financial support with charity insurance coverage, etc.), homeless patients have higher rates of inappropriate ED visits with relatively higher EDRV rates as compared to non-homeless patient populations [14, 15]. Therefore, it is important to determine whether EDRV should be considered one of the patient care quality measurements, specifically among the homeless patient population.

Identifying risks predictive of EDRV among homeless patient populations will help us better understand the pattern of EDRV. Meanwhile, recognizing the differences between homeless and non-homeless patient populations will avoid unnecessary/ineffective interventions and provide a foundation for future, more efficacious interventions. Hence, we aim to 1) identify the differences between homeless and non-homeless patient populations, especially among patients with 72-h EDRV; and 2) recognize risks predictive of such EDRV.

\section{Materials and Methods}

\section{Study design and setting}

This is a retrospective single-center observational study. Data 
were collected via local hospital homeless registry which was prospectively collected. Investigators reviewed all ED data from September 1, 2015 through June 30, 2016. The study hospital (JPS Health Network) is a publicly funded, county hospital and an urban tertiary referral center serving approximately 2 million populations locally. The hospital ED has annual patient visits of more than 120,000. The local Institutional Review Board approved this study.

\section{Participants}

We included all patients presenting to the study hospital ED that were: 1) discharged; 2) left without being seen (LWBS); 3) left against medical advice (AMA); and 4) eloped during the initial ED visit. We excluded: 1) patient $<18$ year old; 2) patients that expired during the initial ED visit; 3) patients admitted to hospital during the initial ED visit; 4) patients transferred to other facilities during the initial ED visit; and 5) prisoners.

\section{Homeless patient population and homeless assisted pro- gram}

We focused on analyzing the ED homeless patient population in this study. Homeless patients were defined as those who met the US Department of Housing and Urban Development (HUD) definition of homelessness at the time of entry into our electronic medical record (EMR) system. Such patients were initially identified via our EMR system and then further paired with the Tarrant County Homeless Management Information System (HMIS) database archived in Fort Worth, TX, USA. In addition to systematically identifying homeless patients, the Care Connection for the Homeless Team (CCHT) at the study hospital had the ability to further identify homeless patients who may have been missed due to data matching issues or did not exist in the HMIS system. For situations where data were not matched between systems, but homeless status was verified by CCHT, patients were entered into the EMR manually. The hospital CCHT program is intended to assist the homeless patient population seeking affordable healthcare locally by 1 ) identifying such patient population; 2) providing financial support (e.g., providing charity healthcare insurance coverage); and 3) arranging primary care physician (PCP) follow-up (e.g., assigning each homeless patient a designated PCP).

\section{ED-related versus unrelated return visits}

Patients who revisited the study ED within $72 \mathrm{~h}$ from the index ED discharge were considered EDRV. Two individual ED physicians, blinded to the study's purpose, reviewed enrolled patient charts to determine whether EDRVs were related or unrelated to the initial ED visits. To resolve discrepancies, a second round of reviews was rendered at least 30 days apart from the previous review. From the second round, physicians only reviewed discrepancy charts. Repeated reviews occurred until a strong level of interrater agreement (kappa $>80 \%$ ) was reached. Once a strong interrater agreement was reached, a third ED physician was consulted to make final decisions concerning the discrepancy of remaining charts.

\section{Different levels of ED utilization}

The New York University ED Algorithm (NYUA) was used to determine different levels of ED utilization. Based on NYUA, four major categories were generated: 1) emergent not avoidable, considered as ED appropriate visits; 2) primary care treatable, defined as care that can be safely provided in a primary care setting without the need for emergent treatment; 3) emergent care needed but preventable/avoidable, defined as patients whose disease conditions can be prevented/avoided if preventive care is received in a timely fashion; and 4) non-emergent. Appropriate ED utilization was considered if patients met the emergent not avoidable category criteria and inappropriate utilization was determined if patients were classified as nonemergent.

\section{Variables}

Patients' general characteristics including age, sex, race/ethnicity, mode of arrival, and insurance type were collected. Mode of arrival was further divided into three categories: 1) private transportation (private car or taxi); 2) healthcare assisted transportation (ambulance ground transportation, flight, and hospital assisted transportation); and 3) public/other transportation (public vehicle, ambulatory, and wheelchair). Insurance type was divided into three categories: 1) private/commercial insurance; 2) government/charity (Medicaid, Medicare, and other national and or local charity insurance plans); and 3) no insurance. Patient condition variables analyzed in this study were: 1 ) whether patient has primary care physician assigned; 2) history of alcohol abuse; 3) history of other substance abuse; and 4) history of psychiatric disorders. Psychiatric disorders included patients with history of depression, anxiety, mania, schizophrenia, schizoaffective disorder, and bipolar disorder. Patient/ED operational variables were 1) patient triage level of acuity using 5-point Emergency Severity Index (ESI); 2) total ED length of stay (LOS); 3) ED medication prescription upon discharge, and 4) return ED dispositions. Return ED dispositions were divided into three categories: 1) significant EDRV disposition deviation, defined as an obvious disposition difference between initial ED visit and return visit (i.e., disposition deviations included hospital admissions, hospital observation, patient expiration during EDRV, or transfer to operating room); 2) non-significant ED disposition deviation (i.e., patients discharged during return visits); and 3) uncertain ED disposition deviation including patients that eloped, left AMA, LWBS, and those transferred to other services or facilities during EDRV.

\section{Outcome measurements}

The primary outcome measurement was 72-h EDRV among 
the homeless patient population. Secondary outcome measurement included a comparison of EDRV patterns between homeless and non-homeless patient populations.

\section{Study protocol}

Enrolled patients in this study were divided into two groups, homeless patients and non-homeless patients. General patient characteristics, patient conditions, patient ED metrics, and patient ED utilization patterns were compared between the two groups. Time-to-EDRV curve was drawn and compared between homeless and non-homeless patients. Risks predictive of EDRV were also analyzed and compared in both groups.

\section{Data analysis}

Kappa test was used for interrater variability analysis with $\kappa$ $>0.8$ indicating a strong level of agreement. A student $t$ test was used for continuous data comparisons and Pearson Chisquare test was used for categorical data comparisons between these two groups. We used multivariate Cox proportional hazard models to examine the relationship between the predictors and the outcomes of interest. Potential independent risks included age, sex, race/ethnicity, mode of arrival, primary care provider assignment, level of acuity, insurance type, history of alcohol abuse, history of substance abuse, history of psychiatric conditions, LOS, and ED medication prescription upon discharge. Time independent categorical variables, which satisfied proportional hazard assumptions, were included in the final model. Equality of strata was used to determine whether the variables should be included in the final model. Risk predictors of EDRV between homeless and non-homeless patient populations were compared with hazard ratio point estimates and a 95\% confidence interval. The results are represented with point estimates and 95\% confidence intervals. Final model was verified for violation of proportional hazard model. Survival analysis was used to find the significant difference in EDRV between homeless and non-homeless patient populations. Time-to-EDRV curve was plotted using Kaplan Meier estimates among homeless patients with different levels of ED utilization as determined by NYUA. All analyses were performed using STATA 14.2 software with P-value $<0.5$ considered statistically significant.

\section{Ethical approval statement}

This study was approved by the John Peter Smith Health Network Institutional Review Board.

\section{Results}

\section{General information}

A total of 63,990 patient encounters were enrolled in this study comprising 5,926 homeless ED visits during the study period. Two rounds of provider reviews were performed to determine whether EDRVs were related to initial ED visits. Interrater agreement was 0.74 (first round, $\mathrm{P}<0.05$ ) and 0.93 (second round, $\mathrm{P}<0.05$ ), indicating a strong level of agreement between providers. A detailed interpretation of patient information is provided in Table 1. Briefly, among all enrolled patients, homeless patients tended to be older, predominately male, arrived more frequently to the ED via healthcare assisted transportation, and more commonly had PCPs assigned with government/charity funded insurance. Patients presenting for these encounters also had a higher incidence of history of alcohol or drug abuse and psychiatric conditions. However, EDRV rates among homeless patients were much higher when compared to those of other patient groups. Table 1 indicates a potential special patient population requiring separate analysis.

\section{EDRV in homeless patient population}

Further analysis was rendered among patients with EDRVs (Table 2). Among all EDRVs, no significant differences occurred in terms of related versus unrelated EDRVs between these two groups. Additionally, ED utilization patterns were similar and less significant EDRV disposition deviations occurred among the homeless patient population compared to those in the non-homeless patient population $(15 \%$ versus $23 \%, \mathrm{P}<0.001)$. After adjusting for all potential risks, a similar hazard ratio occurred regardless of homeless condition, indicating similar EDRV patterns in general (Table 3).

\section{Time-to-EDRV curve}

To identify potential EDRV pattern differences between homeless and non-homeless populations, patients with EDRV were included in the time-to-event analysis using Kaplan-Meier survival estimates (Fig. 1a). The median time interval of EDRV in homeless patients was $31 \mathrm{~h}$ (interquartile range (IQR) 20 - 49) and the median time interval of EDRV in non-homeless patients was $32 \mathrm{~h}$ (IQR 18 - 50). Our finding consistently showed similar EDRV patterns between homeless and non-homeless patient populations. Furthermore, similar Time-to-EDRV curves were shown regardless of different levels of ED utilization among homeless patients (Fig. 1b).

\section{Discussion}

Homeless patients are considered a special patient population tending to have higher ED utilization, hospital admission, and mortality rates $[16,17]$. Such findings are quite controversial in the current literature [18]. Our study findings indicate that the homeless patient population differs from other patient populations. However, their EDRV patterns are quite similar. Though significantly high EDRV rates occurred in the homeless patient 
Table 1. General Information of Study Population

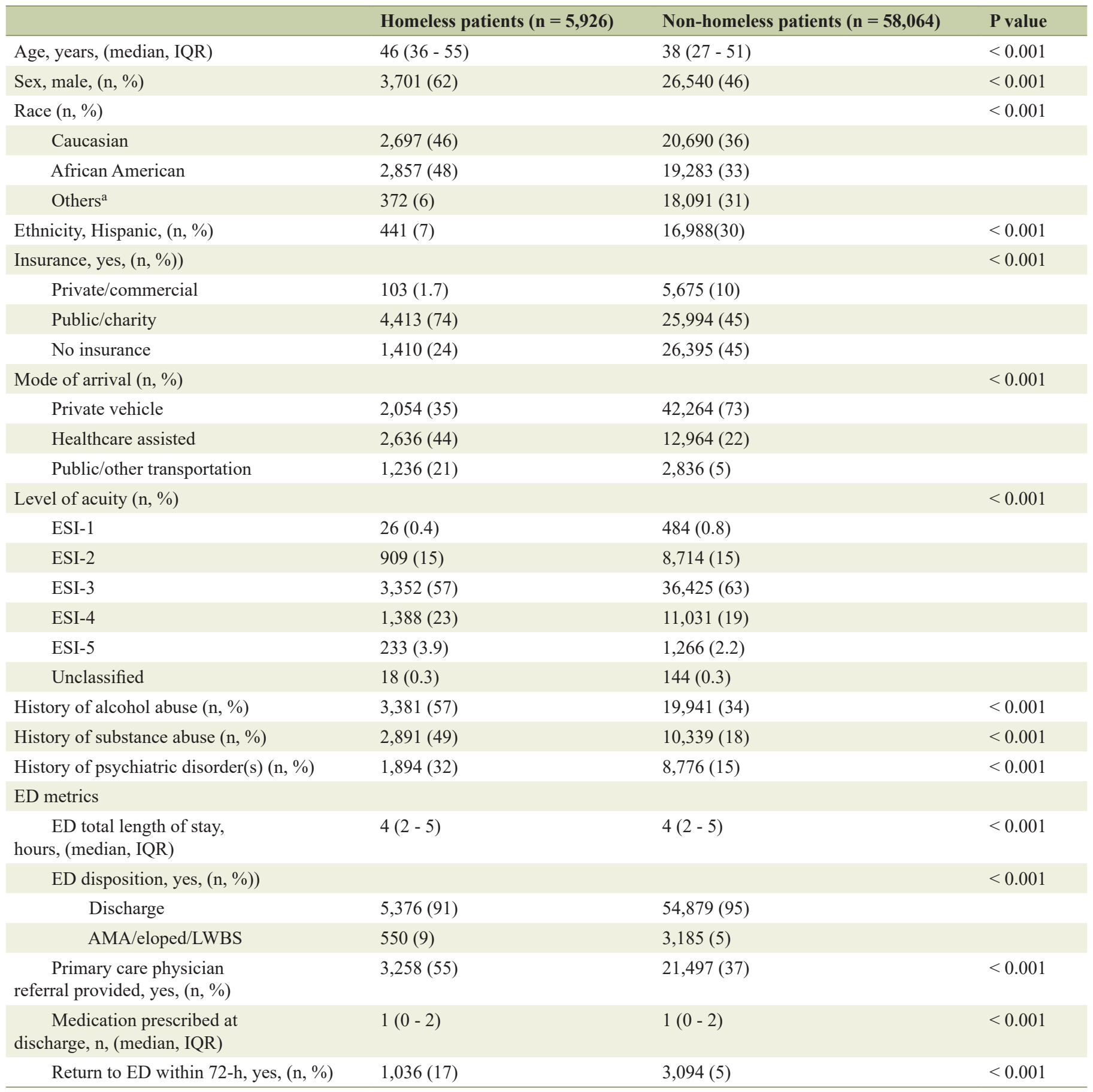

IQR: interquartile range; n: number; ESI: Emergency Severity Index; AMA: against medical advice; LWBS: left without being seen. aOthers including American Indian, Alaska Native, Asian, Native Hawaiian or other Pacific Islander, patient refused, or unknown.

population, their ED management seems to be reasonable with no significant disposition deviations. Similar risks predicting EDRV occurred regardless of homeless status. Unfortunately, though we intend to assist homeless patients with affordable healthcare by providing charity insurance and dedicated PCP assignments, these interventions did not positively impact
EDRV in this population. A more robust intervention might be required for reducing inappropriate ED utilization in the homeless cohort.

Our study findings compliment the current literature. We support that 72-h EDRV should not be included as an ED patient quality measurement, specifically in homeless patients. 
Table 2. Comparison of 72-H Emergency Department Returns in Homeless Patients

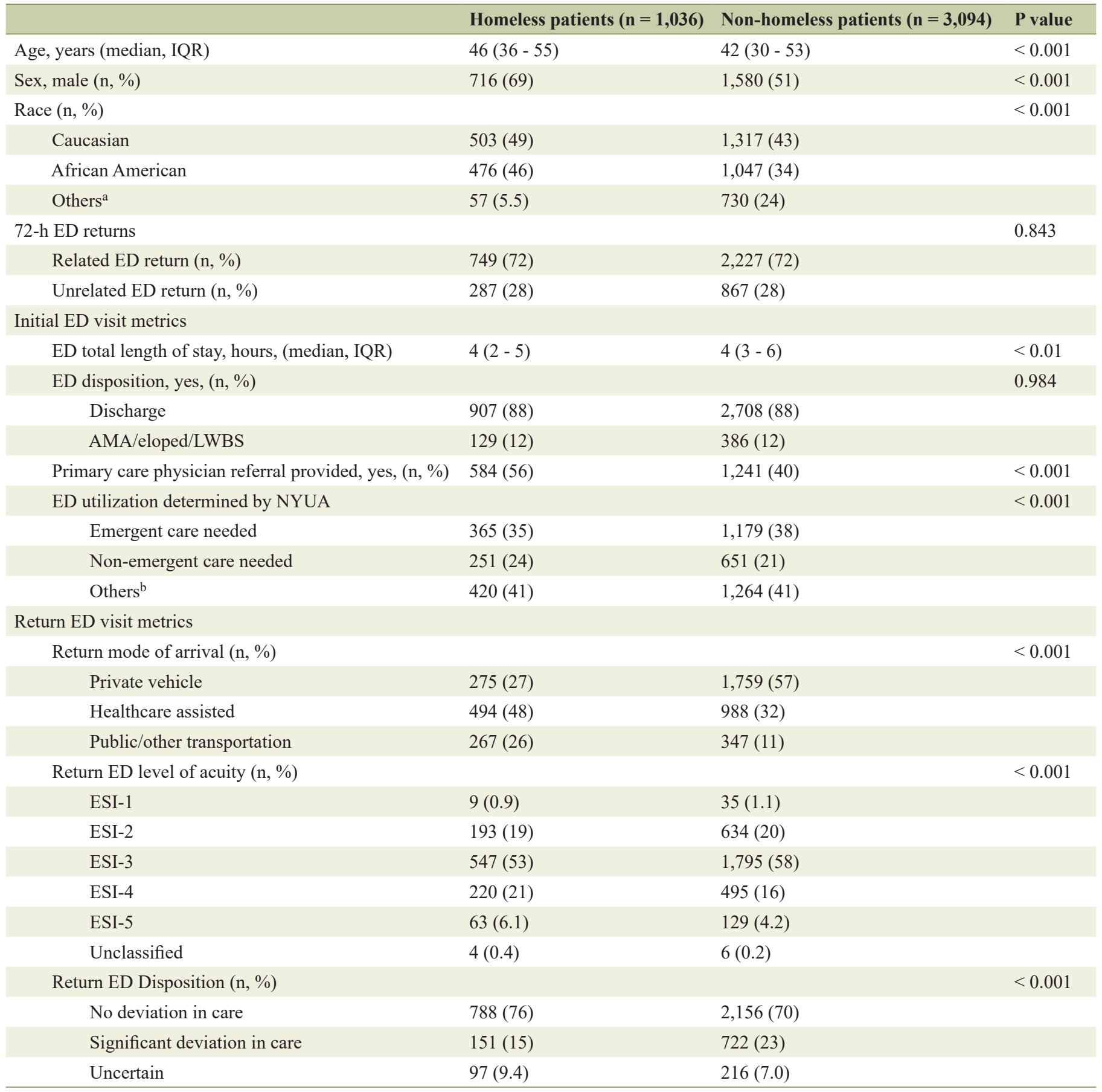

IQR: interquartile range; n: number; AMA; against medical advice; LWBS: left without being seen; NYUA: New York University ED Algorithm; ESI: Emergency Severity Index. ${ }^{a}$ Others including American Indian, Alaska Native, Asian, Native Hawaiian or other Pacific Islander, patient refused, or unknown. ${ }^{b}$ Others including emergent/primary care treatable, emergent (ED care needed, preventable/avoidable), or unclassified one which referred to patients with injury, drug, psychiatric, or alcohol-associated ED visits.

We identified similar risk patterns of EDRV in homeless patients despite the return visit relationship to the initial ED visit. Homeless patient ED utilization patterns are quite similar in comparison to non-homeless patient populations. Therefore, further interventions aimed at reducing EDRV can be rendered without specifying homeless as a unique patient condition. With such evidence, it seems more appropriate that interventions prioritize resolution of psychosocial issues over providing affordable healthcare among the homeless patient population. 
Table 3. Comparison of Risks Predictive of ED 72-H Returns in Homeless Patients

\begin{tabular}{|c|c|c|}
\hline & Homeless patients adjusted hazard ratios & Non-homeless patients adjusted hazard ratios \\
\hline & 95\% CI (LL, UL) & 95\% CI (LL, UL) \\
\hline Sex (female) & $0.72(0.62,0.84)$ & $0.88(0.82,0.95)$ \\
\hline \multicolumn{3}{|l|}{ Mode of arrival } \\
\hline Private vehicle & Reference & Reference \\
\hline \multicolumn{3}{|l|}{ Insurance } \\
\hline Commercial insurance & Reference & Reference \\
\hline Public/charity insurance & $1.58(0.84,3.00)$ & $1.59(1.37,1.85)$ \\
\hline No insurance & $1.27(0.67,2.44)$ & $1.10(0.95,1.29)$ \\
\hline History of psychiatric disorder(s) (yes) & $1.40(1.22,1.62)$ & $1.50(1.37,1.64)$ \\
\hline \multicolumn{3}{|l|}{ Initial ED disposition } \\
\hline Discharged & Reference & Reference \\
\hline AMA/eloped/LWBS & $1.57(1.26,1.94)$ & $2.56(2.27,2.88)$ \\
\hline
\end{tabular}

Cl: confidence interval; LL: lower limit; UL: upper limit; AMA: against medical advice; LWBS: left without being seen.

Our study confirms that homeless patients should be treated as a special patient population, as they differ significantly in terms of patient general characteristics. However, the overall 72-h EDRV rate from our study falls into the same range of EDRV rates across the nation [15]; therefore, raising the question as to whether or not $72-\mathrm{h}$ is an adequate time interval for identifying poor patient care outcomes. Recent studies have identified homeless patients with longer time intervals (e.g., 9 days, 30 days) and suggested that longer time intervals of EDRV are required to realize meaningful healthcare quality measurement analyses $[19,20]$. Others recommend tracking EDRV relative to patients with specific disease patterns (e.g., traumatic brain injury, abdominal pain, heart problem, etc.) rather than 72-h EDRV as a marker for healthcare quality monitoring $[7,8]$. Homeless patients, in general, having significant psychosocial risks and higher ED utilization in the setting of less severe medical concerns $[18,21]$, might require longer time intervals from the index ED discharge to more accurately indicate their appropriate healthcare needs. Future research will be focused on interventions to reduce such EDRV among
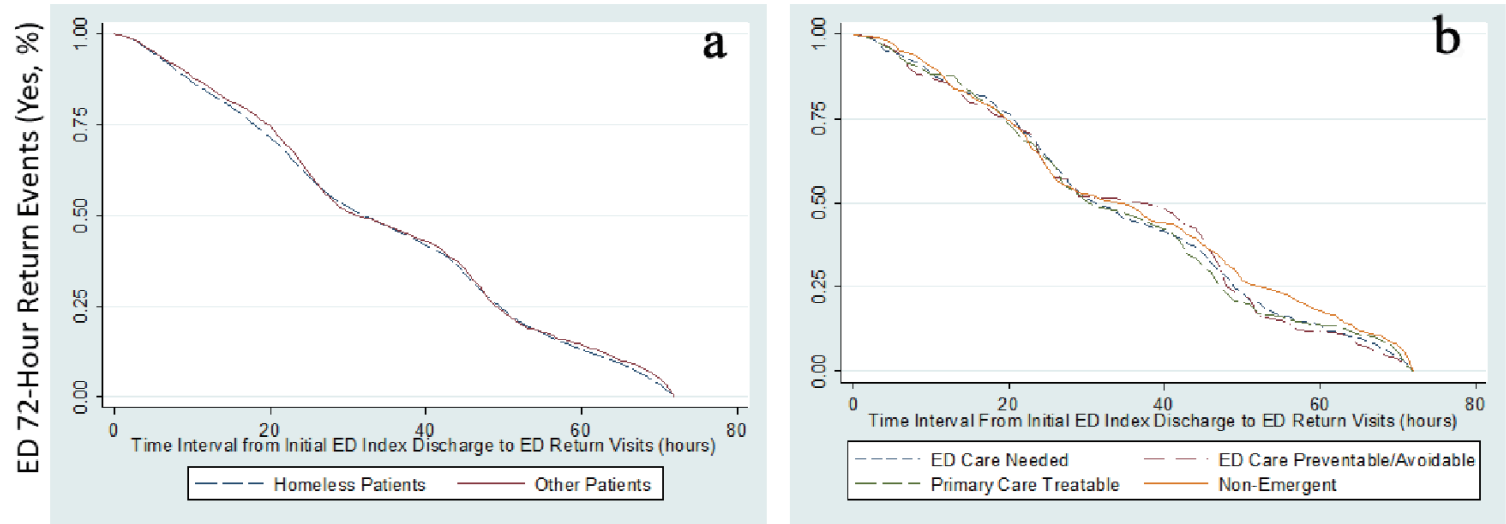

Figure 1. Time interval from ED index discharge to ED return cures using Kaplan-Meier survival analysis. (a) Time interval from ED index discharge to ED return curve comparisons between homeless patients and other (non-homeless) patients. (b) Time interval from ED index discharge to ED return curve comparisons among homeless patients with different classifications of ED utilization as determined by New York University ED Algorithm. 
homeless patients.

\section{Limitations}

This is a retrospective single-center observation study with potential patient selection, missing data, and incorrect information biases. Patients with missing information were excluded in this study which could affect study findings. However, this study was a secondary data analysis from local homeless registry whose data were prospectively collected with very few missing information. Secondly, we were not able to identify all homeless patients despite a robust dual systems utilization process. We were also unable to identify patients whose 72-h ED returns were from other hospitals. Thirdly, as risks predictive of EDRV are multifactorial, we may have overlooked other potential risks in our analysis. Patient 72-h EDRV may not be a suitable time interval for a meaningful healthcare quality measurement, an extended time interval EDRV should be rendered in future studies. The study hospital has already established outreach homeless assistance programs which enroll a significant number of homeless patients as one of the missions of our publicly funded county hospital. Most homeless patients in our region choose the study hospital as their medical home. This explains the fact that our homeless patients have high PCP assignment and charity insurance coverage rates. Though some interventions might not be reproducible, our study validated the other studies' findings that access to PCP alone seems inadequate to reduce ED utilization in this special patient population [14]. A special home clinic for homeless patients near their shelter locations was implemented in the study hospital and research identifying the efficacy of that intervention will be reported in the future.

\section{Conclusions}

Risk patterns of EDRV and time to EDRV are similar between homeless and non-homeless patient populations. Though EDRV rates are significantly high among homeless patients, their return management seems appropriate indicating that 72-h EDRV might not be an optimal healthcare quality measurement for homeless patients.

\section{Financial Disclosure}

Authors have no financial disclosures to report.

\section{Conflict of Interest}

No potential conflicts of interest exist for all authors.

\section{Author's Contribution}

HW conceived the study and designed the protocol. HW, HK,
$\mathrm{CH}$, and RM performed the study and data collection. HW, RDR, SMT, and NRZ performed the data analysis. HW, RDR, JLP, JK, and NRZ drafted the article, and all authors contributed substantially to this study. HW takes responsibility for the paper as a whole.

\section{References}

1. Keith KD, Bocka JJ, Kobernick MS, Krome RL, Ross MA. Emergency department revisits. Ann Emerg Med. 1989;18(9):964-968.

2. Lerman B, Kobernick MS. Return visits to the emergency department. J Emerg Med. 1987;5(5):359-362.

3. McCusker J, Ionescu-Ittu R, Ciampi A, Vadeboncoeur A, Roberge D, Larouche D, Verdon J, et al. Hospital characteristics and emergency department care of older patients are associated with return visits. Acad Emerg Med. 2007;14(5):426-433.

4. Shy BD, Shapiro JS, Shearer PL, Genes NG, Clesca CF, Strayer RJ, Richardson LD. A conceptual framework for improved analyses of 72-hour return cases. Am J Emerg Med. 2015;33(1):104-107.

5. Pham JC, Kirsch TD, Hill PM, DeRuggerio K, Hoffmann B. Seventy-two-hour returns may not be a good indicator of safety in the emergency department: a national study. Acad Emerg Med. 2011;18(4):390-397.

6. Cheng J, Shroff A, Khan N, Jain S. Emergency department return visits resulting in admission: do they reflect quality of care? Am J Med Qual. 2016;31(6):541-551.

7. Ganti L, Conroy LM, Bodhit A, Daneshvar Y, Patel PS, Ayala S, Kuchibhotla S, et al. Understanding why patients return to the emergency department after mild traumatic brain injury within 72 hours. West J Emerg Med. 2015;16(3):481-485.

8. Chan AH, Ho SF, Fook-Chong SM, Lian SW, Liu N, Ong ME. Characteristics of patients who made a return visit within 72 hours to the emergency department of a Singapore tertiary hospital. Singapore Med J. 2016;57(6):301306.

9. Pereira L, Choquet C, Perozziello A, Wargon M, Juillien G, Colosi L, Hellmann R, et al. Unscheduled-return-visits after an emergency department (ED) attendance and clinical link between both visits in patients aged 75 years and over: a prospective observational study. PLoS One. 2015;10(4): e0123803.

10. $\mathrm{Ku} \mathrm{BS}$, Scott KC, Kertesz SG, Pitts SR. Factors associated with use of urban emergency departments by the U.S. homeless population. Public Health Rep. 2010;125(3):398-405.

11. Khandor E, Mason K, Chambers C, Rossiter K, Cowan L, Hwang SW. Access to primary health care among homeless adults in Toronto, Canada: results from the Street Health survey. Open Med. 2011;5(2):e94-e103.

12. Gabrielian S, Yuan AH, Andersen RM, Rubenstein LV, Gelberg L. VA health service utilization for homeless and low-income Veterans: a spotlight on the VA Supportive Housing (VASH) program in greater Los Angeles. Med Care. 2014;52(5):454-461. 
13. Department of Veterans Affairs. Health Care for Homeless Veterans program. Final rule. Fed Regist. 2015;80(84):24819-24821.

14. Hategan A, Tisi D, Abdurrahman M, Bourgeois JA. Geriatric homelessness: association with emergency department utilization. Can Geriatr J. 2016;19(4):189-194.

15. Shy BD, Kim EY, Genes NG, Lowry T, Loo GT, Hwang $\mathrm{U}$, Richardson LD, et al. Increased identification of emergency department 72-hour returns using multihospital health information exchange. Acad Emerg Med. 2016;23(5):645-649.

16. Nusselder WJ, Slockers MT, Krol L, Slockers CT, Looman CW, van Beeck EF. Mortality and life expectancy in homeless men and women in Rotterdam: 20012010. PLoS One. 2013;8(10):e73979.

17. Mackelprang JL, Qiu Q, Rivara FP. Predictors of emergency department visits and inpatient admissions among homeless and unstably housed adolescents and young adults. Med Care. 2015;53(12):1010-1017.

18. Wang H, Nejtek VA, Zieger D, Robinson RD, Schrader $\mathrm{CD}$, Phariss $\mathrm{C}, \mathrm{Ku} \mathrm{J}$, et al. The role of charity care and primary care physician assignment on ED use in homeless patients. Am J Emerg Med. 2015;33(8):1006-1011.

19. Lam CN, Arora S, Menchine M. Increased 30-day emergency department revisits among homeless patients with mental health conditions. West J Emerg Med. 2016;17(5):607-612.

20. Rising KL, Victor TW, Hollander JE, Carr BG. Patient returns to the emergency department: the time-to-return curve. Acad Emerg Med. 2014;21(8):864-871.

21. Hodgson KJ, Shelton KH, van den Bree MB, Los FJ. Psychopathology in young people experiencing homelessness: a systematic review. Am J Public Health. 2013;103(6):e24-37. 